\title{
Fetal Lungs of Tenascin-C-Deficient Mice Grow Well, but Branch Poorly in Organ Culture
}

\author{
Matthias Roth-Kleiner, Emilio Hirsch, and Johannes C. Schittny \\ Institute of Anatomy, University of Bern, Bern; Division of Neonatology, Centre Hospitalier Universitaire Vaudois, \\ Lausanne, Switzerland; and Max-Planck-Institute of Biochemistry, Martinsried, Germany
}

\begin{abstract}
Tenascin-C (TNC) is a multidomain extracellular matrix protein that contributes to organogenesis and tumorgenesis. To elucidate its developmental function in the context of TNC deficiency, lung lobes of TNC null mice were obtained at Embryonic Days E11.5 and E12.5 and cultured for $3 \mathrm{~d}$. In lung explants of homozygote TNC-deficient embryos (E12.5) the number of future airway branches was reduced by $36 \%$ as compared with wild-type. In heterozygote explants only half of the reduction (18\%) was observed. No significant alteration, neither of the explant growth nor of the pattern of airway branching, was noticed in TNC-null explants. However, the terminal endbuds of the transgenic explants were enlarged. The results are supported by a morphologic investigation at Postnatal Day P2, where the airspaces of TNC-deficient lungs appeared larger than in wild-type lungs. Taken together, our results represent the first developmental phenotype of TNC-null mice. We conclude that TNC takes part in the control of fetal lung branching, and that not only the presence of TNC but also its amount is important. Because TNC is predominantly expressed at the growing tip of the future airways, we hypothesize that TNC promotes the penetration into the surrounding mesenchyme and the branching of the growing airways.
\end{abstract}

\section{Lung Development}

Mouse lung development comprises six different stages. Organogenesis starts with the embryonic period (Embryonic Days E9.5-E12). The lung anlage forms by a ventral outpouching of the foregut at the caudal end of the laryngotracheal sulci. The two formed lung buds, will give rise to the main bronchi (1). Cranial of the lung buds, the prospective trachea, and esophagus form by separation from the foregut (2). The prospective main bronchi divide dichotomously and invade the surrounding mesenchyme forming the future bronchi. Looking like a primitive gland, the developing lung enters the pseudoglandular stage (E12-E16.5). Most of the branching morphogenesis takes place during this stage. Epithelial differentiation starts and the future bronchi and bronchioli begin to acquire their smooth muscle layer. The canalicular stage (E16.5-E17.5) is characterized by a "canalization" of the future lung parenchyma by capillaries. Branch-

(Received in original form November 22, 2002 and in revised form July 18, 2003) Address correspondence to: Johannes C. Schittny, Institute of Anatomy, University of Bern, Buehlstrasse 26, CH-3000 Bern 9, Switzerland. E-mail: schittny@ana.unibe.ch

Abbreviations: embryonic, E; postnatal, P; phosphate-buffered saline, PBS; Tenascin-C, TNC; transferase-mediated deoxyuridine triphosphate nick end labeling, TUNEL.

Am. J. Respir. Cell Mol. Biol. Vol. 30, pp. 360-366, 2004

Originally Published in Press as DOI: 10.1165/rcmb.2002-0266OC on August 6, 2003

Internet address: www.atsjournals.org ing comes to completion, the formation of the air-blood barrier starts, and the epithelial type II cells produce the first surfactant. During the saccular stage (E17.5-P4) terminal clusters of widened spaces (saccules) are formed in the peripheral airways. The capillary network surrounding the saccules condenses and forms two capillary layers located directly underneath both septal surfaces. As a result the septa now contain two sheets of capillary layers separated by a core of connective tissue. After birth, during the phase of alveolarization (P4-P14), the alveoli are formed by lifting off of secondary alveolar septa from the existing primary septa. The double capillary layer of the immature alveolar septa is reduced to a single capillary layer during the phase of microvascular maturation (P14-P21) $(3,4)$.

\section{Tenascin-C}

Tenascin-C (TNC) belongs to a family of at least five large extracellular matrix glycoproteins (tenascin-C, tenascin-R, tenascin-W, tenascin-X, and tenascin-Y). TNC is detectable only in small amounts in normal adult tissues. Larger amounts are expressed during development, as well as in adults during tissue repair and remodeling, especially during neovascularization, wound healing, and tumorgenesis $(5,6)$. Whereas tenascin- $\mathrm{R}$ is highly restricted to the central nervous system, the expression of TNC and tenascin-X is frequently reciprocal. In skin, tendons, ligaments, heart, uterus, placenta, aorta, skeletal muscle, and nerves, expression of tenascin-X prevails. But in fetal brain and spleen, adult spinal cord, kidney, and in lung, TNC appears to be leading $(7,8)$. Tenascin-W, the newest member of the tenascin family, was identified in zebra fish and colocalizes with TNC in neural crest (9). TN-Y represents the avian homolog to mammalian tenascin- $X$ and has been identified in heart and skeletal muscle and in the connective tissue of skeletal muscle (10).

The expression of TNC is upregulated by different factors like transforming growth factor $\beta 1$, fibroblast growth factor, keratinocyte growth factor, nerve growth factor, and mechanical stress. Glucocorticoids downregulate TNC expression. TNC contributes to the regulation of cell proliferation, migration, growth, and survival during organogenesis and tissue repair. Its developmental role is underlined by its accumulation in the vicinity of actively growing epithelia-meaning in their basement membranes and their surrounding mesenchyme (11-13).

\section{Tenascin-C during Lung Development}

Throughout prenatal lung development TNC accumulates in the basement membranes and mesenchyme surrounding 
the branching and growing tips of the bronchial tree. In chicken TNC is expressed by epithelial cells (14), whereas in mice and rats it is predominantly expressed by mesenchymal cells at the sites of branching $(3,15,16)$. Perturbation of fetal lung organ cultures with functional anti-TNC antibodies or bacterially expressed $\mathrm{TNC}$ fragments resulted in a reduction of the number of branches. The growth of these explants was not altered (16, and J.C.S., unpublished results).

Postnatally TNC is expressed at the tips of the newly forming septa during the phase of alveolarization. In 3D these tips represent the alveolar entrance rings (alveolar mouths). In addition, they contain elastic fibers, collagen, and smooth muscle cells. It is believed that they have to bear mechanical forces. In later stages no TNC expression was observed anymore (16).

\section{Tenascin-C Knockout Mice}

According to the described developmental role of TNC, it was rather surprising when Saga and coworkers presented a homozygous TNC-deficient mouse without an apparent phenotype (17). Forsberg and colleagues repeated this experiment obtaining the same result by introducing an independently produced TNC-null mouse (18). Both mouse strains showed no significant difference from wild-type with respect to size, life span, or fertility. A brief histologic examination of the principal organ systems including lung did not reveal any further phenotype. More detailed investigations were necessary to recognize subtle phenotypes like abnormal behavior (19), reduced haemopoietic activity of bone marrow cells (20), decreased reaction on suture wounds of cornea (21), a reduced proliferation of mesangial cells, and abnormal restoration after habu-snake venom-induced glomerulonephritis (22) (for review see Ref. 11).

Due to the complexity of lung development, a lung phenotype may easily be overseen or an early developmental phenotype may be rescued during later stages of development. Therefore, we investigated lung branching morphogenesis in TNC null mice of Forsberg and associates (18) using lung organ cultures.

\section{Materials and Methods \\ Animals}

The TNC-null mouse strain of Forsberg and coworkers was used (18). Wild-type littermates and $129 / \mathrm{Sv}$ mice were used as controls. Fetuses (12.5 d old) were obtained from heterozygote breeding; 11.5-d-old fetuses were obtained from homozygote breeding. Phenotyping was done according to Forsberg and colleagues (18). Handling of the animals before and during the experiments, as well as the experiments themselves, were approved and supervised by the Swiss Agency for the Environment, Forests and Landscape and the Veterinary Service of the Canton of Bern.

\section{Lung Organ Culture}

Fetal mouse lungs were obtained at Day E11.5 or E12.5 under sterile conditions and cultured as described previously (23). At Day E12.5 lung lobes were separated in phosphate-buffered saline (PBS; $127 \mathrm{mM} \mathrm{NaCl}, 10 \mathrm{mM} \mathrm{Na} \mathrm{HPO}_{4}, \mathrm{pH}$ 7.4) and cultured individually at the air-culture medium interface on a floating filter (TSTP Isopore filter, $13 \mathrm{~mm}$ diameter, $3 \mu \mathrm{m}$ pore size; Millipore,

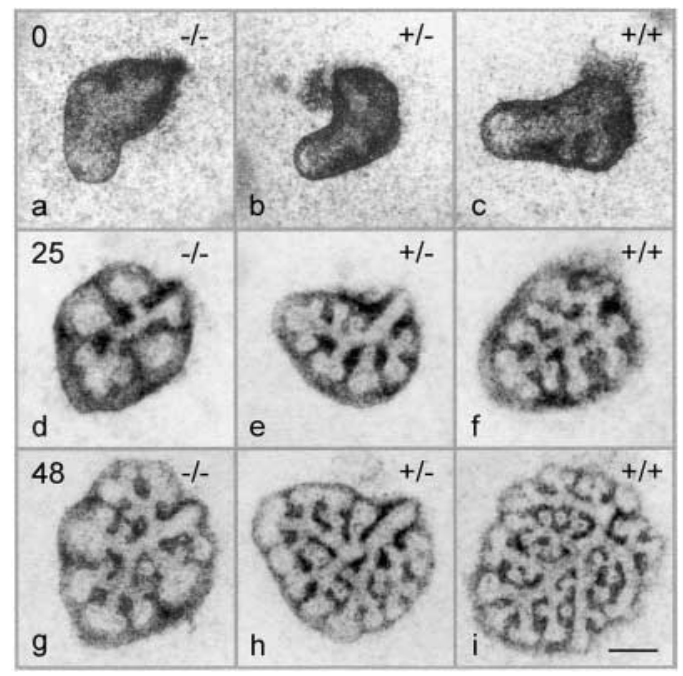

Figure 1. Lung organ culture. Lung lobes were obtained from TNC-deficient ( $a, d, g ;-/-)$, heterozygote $(b, e, h ;+/-)$, and wildtype fetuses $(c, f, i ;+/+)$ on Embryonic Day E12.5. As compared with wild-type, TNC-deficient and heterozygote explants developed a smaller number of branches. Organ explant growth appeared unaltered, but the terminal endbuds looked larger. The images were taken at the start of the organ culture (Hour $0 ; a, b$, $c)$, after $25 \mathrm{~h}(d, e, f)$ and $48 \mathrm{~h}(g, h, i)$. Bar: $0.5 \mathrm{~mm}$.

Bedford, MA) in a 24-well plate (Falcon-Becton Dickinson, Lincoln Park, NJ) at $37^{\circ} \mathrm{C}$ in $5 \% \mathrm{CO}_{2} /$ air. Lungs obtained at E11.5 were explanted undissected. The medium, Dulbecco's modified eagle medium (GibcoBRL-Life Technologies, Basel, Switzerland), was enriched with $10 \%$ fetal calf serum (GibcoBRL), $5 \mu \mathrm{g} / \mathrm{ml}$ bovine insulin (Sigma Chemicals Co., St. Louis, MO), 2 mM glutamine (Sigma), $75 \mu \mathrm{g} / \mathrm{ml}$ streptomycin (Sigma), and $100 \mu \mathrm{g} / \mathrm{ml}$ penicillin (Sigma). Three explants were transferred onto each filter and the filters were placed in a well containing $350 \mu \mathrm{l}$ medium. The distance between the three explants was large enough to ensure that the tissues did not touch during growth. The explants were fed daily by adding $200 \mu \mathrm{l}$ of fresh medium per well. Images of the explants were taken twice a day using an inverted microscope (Diaphot-TMD; Nikon, Tokyo, Japan).

\section{Statistical Evaluations}

As a measure for number of branches, the number of terminal endbuds was counted on micrographs as shown in Figure 1. The growth (volume \%) was estimated by area measurement of the projected organ pieces. The growth was calculated by dividing the measured area by the area of the explant at the start of the experiment and multiplying this result by 100 . We may take these numbers as an estimation of the volume \% of the growth, because the quotient of the diameter and the thickness of the individual explant were constant, especially as compared between different explants at one time point, but also between explants at different time points.

The size of the terminal endbuds was measured as follows. For all explants obtained at Day E11.5 branching of the endbuds was followed individually in the time interval of $15-40 \mathrm{~h}$ after explantation. At time point $40 \mathrm{~h}$ the endbuds were grouped into the categories "no branching," "branched once," and "branched twice," and their size was measured on $\times 23$ enlarged prints using a graphics 


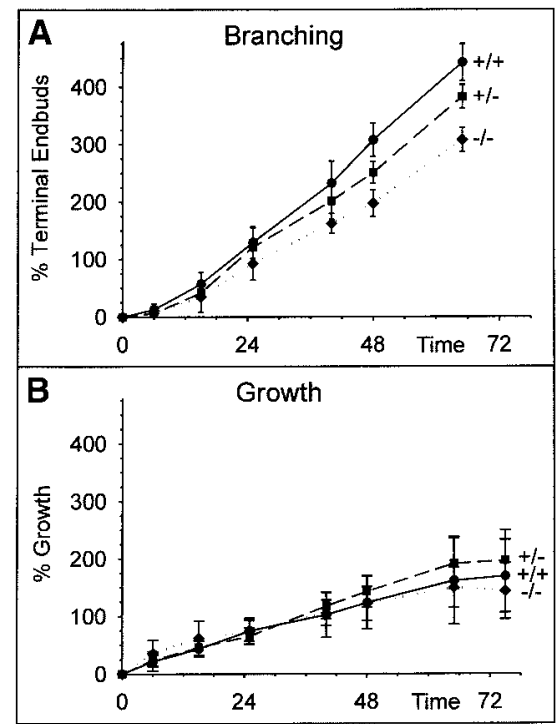

Figure 2. Statistical evaluation of the lung organ culture results (Figure 1). The number of branches was assessed by counting the terminal endbuds $(a)$. After $48 \mathrm{~h}$ a significant difference between wild-type $(+/+)$, heterozygote $(+/-)$, and homozygote $(-/-)$ TNC-deficient explants was observed. Area measurements of the projected organ pieces were used to estimate the growth (volume $\%)$. Even after $3 \mathrm{~d}$ no significant differences were observed $(b)$. Bars include the standard deviation.

tablet of a pentium base personal computer system and the software SigmaScan (SPSS Inc., Chicago, IL). A histogram of the categories was drawn and the mean size of terminal endbuds was calculated separately for homozygote null, heterozygote, and wildtype explants.

For the estimation of the size of the airspaces at Day P2, central paraffin sections of three TNC-null and three wild-type left lungs were photographed in a meandering pattern covering $\sim 50 \%$ of the section (one section per lung). The mean volume-weighted airspace volume was estimated as described by Howard and Reed (24). Briefly, airspaces were sampled for measuring using a grid of points. The length of an isotopic line $\left(l_{0}\right)$ passing through the sampling point that lies within the airspace transect was measured for every selected airspace. The mean volume-weighted airspace volume was estimated from

$$
\hat{V}_{v}=\frac{\pi}{3} \cdot \bar{I}_{0}^{3}=\frac{\pi}{3 \cdot n} \sum_{i=1}^{n} I_{0, i}^{3}
$$

where $n$ is the total number of point-sampled linear intercepts and $l_{0, i}{ }^{3}$ is the cube of the $i$ th point-sampled intercept length. Because we used this estimation only for a verification of our morphologic observation, the ratio between homozygote TNC-deficient and wild-type animals is given only.

For all experiments significance was determined using the Student's $t$-test. $P<0.05$ was defined as "significant" and $P<0.001$ as "highly significant."

\section{Transferase-Mediated dUTP Nick End Labeling Assay and Cell Proliferation}

Transferase-mediated dUTP nick end labeling (TUNEL) assay and the immunoperoxidase staining was performed on $10-\mu \mathrm{m}$ cryostat

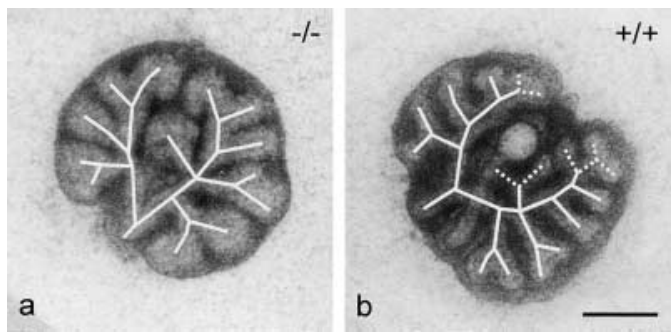

Figure 3. Branching pattern. The branching pattern of TNC null $(a,-/-)$ and wild-type $(b,+/+)$ explants obtained at E11.5 were compared. No difference in the branching pattern (solid lines) was observed. Explants are shown after $30 \mathrm{~h}$ in culture. Additional branches of the wild-type lung are labeled with dotted lines. Bar: $0.5 \mathrm{~mm}$.

cross-sections of lung explants that were cultured for $54 \mathrm{~h}$ as described by Schittny and coworkers $(25,26)$. Briefly, after digestion with $5 \mu \mathrm{g} / \mathrm{ml}$ Proteinase $\mathrm{K}\left(21^{\circ} \mathrm{C}, 10 \mathrm{~min}\right)$ cryosections were incubated with terminal transferase reaction solution, containing $9 \mathrm{mM}$ digoxigenin-11-dUTP and $0.165 \mathrm{U} / \mathrm{ml}$ enzyme (Boehringer Mannheim, Mannheim, Germany) for $50 \mathrm{~min}$ at $37^{\circ} \mathrm{C}$. The incorporated digoxigenin was detected with anti-digoxigenin (rhodamine-antidigoxigenin from Boehringer Mannheim). Proliferating cells were labeled using monoclonal antibody MIB-5 (Immunotech, Marseilles, France) $(27,28)$, which was diluted 1:50 in Tris-buffered saline/bovine serum albumin, containing 1\% normal goat serum. Endogen peroxidases were quenched by a treatment with $3 \%$ $\mathrm{H}_{2} \mathrm{O}_{2}(30 \%)$ in methanol two times for $20 \mathrm{~min}$. First antibody was detected using biotinylated, affinity-isolated goat anti-mouse globulin (diluted 1:200 in Tris-buffered saline; Dakopatts, Glostrup, Denmark), followed by the visualization with avidin-biotin peroxidase complex (ABC; Dakopatts) using diaminobenzidine as substrate.

\section{Morphology}

Lungs were prepared according to Schittny and coworkers (25). Briefly, at Postnatal Day P2 the pulmonary blood vessels were perfused with PBS containing $5 \mathrm{U} / \mathrm{ml}$ heparin, $10 \mathrm{mg} / \mathrm{ml}$ procaine, and $10 \mathrm{mM}$ EDTA (Fluka Chemie AG, Buchs, Switzerland), and the airspaces were filled with PBS containing $4 \%$ freshly prepared paraformaldehyde (Merck, Darmstadt, Germany) at a constant pressure of $20 \mathrm{~cm}$ water column. At this pressure, the lung reaches roughly its midrespiratory volume. To prevent a recoiling of the lung, the pressure was maintained during fixation of $1 \mathrm{~h}$. After three washes in PBS, followed by a graded series of ethanol and by three changes of Histoclear (Life Science International, Frankfurt, Germany), lungs were embedded in paraffin (Histosec; Merck) at $60^{\circ} \mathrm{C}$. Under all conditions no recoil of the lungs was observed. 3.5-5 $\mu \mathrm{m}$ sections were cut, transferred onto silanized (aminopropyl-trimethoxy-silane) microslides, air-dried overnight at $37^{\circ} \mathrm{C}$, dewaxed in Histoclear, and stained with hematoxylin and eosin.

\section{Volumetry}

After fixation the five lobes were separated and their volumes were measured by water displacement (29). The total lung volume was calculated from the five lobar volumes. The specific lung volume was defined as lung volume over body weight (30). 


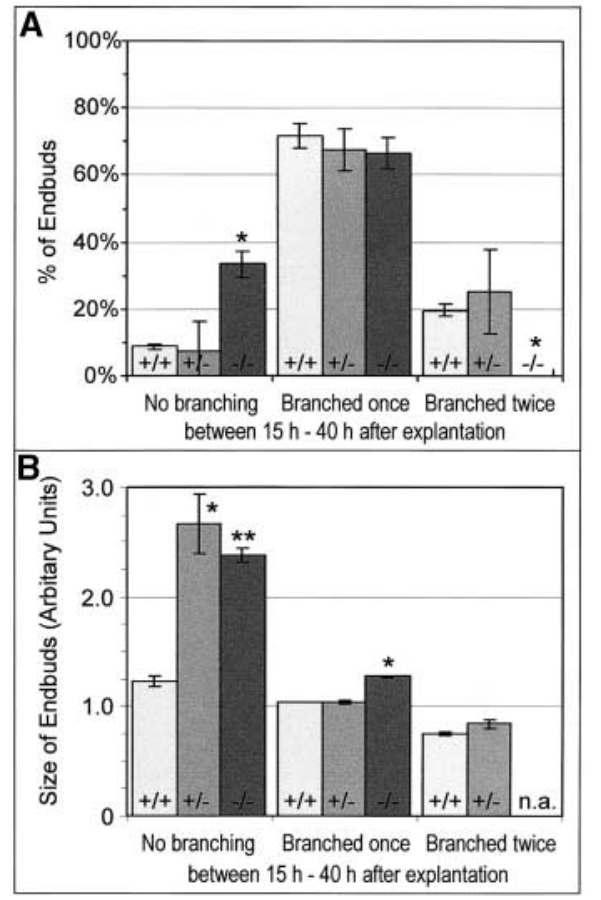

Figure 4. Morphometry of terminal endbuds. We determined how often each individual endbud branched between 15 and $40 \mathrm{~h}$ in culture $(a)$ and measured its size $40 \mathrm{~h}$ after the lungs were explanted $(b)$. The number of terminal endbuds significantly differed between homozygote $(-/-)$ TNC-deficient lung explants and wildtype $(+/+)$ or heterozygote $(+/-)$ ones in the "no branching" $(P=0.003)$ and the "branched twice" $(P=0.028)$ groups $(a)$. As compared with wild-type the size of the endbuds was significantly enlarged for heterozygote $(P=0.004)$ and homozygote-null explants $(P=0.001)$ in the "no branching" group and for homozygote-null explants $(P=0.022)$ in the "branched once" group $(b)$. Some of the data of the heterozygote explants did not reach significance due to the short interval of observation. There were no endbuds found which branched twice in the homozygote null explants. $* P \leqslant 0.05 ; * * P \leqslant 0.001$; n.a., not applicable. Bars include standard errors.

\section{Results}

\section{Lung Organ Culture}

Lung organ cultures were used to compare branching morphogenesis of TNC null and wild-type lungs. Fetal lungs of TNC-deficient (18) and of wild-type embryos were explanted at Days E11.5 or E12.5 and cultured for at least $3 \mathrm{~d}$ (Table 1). As wild-type controls $129 \mathrm{SV}$ mice were used at Day E11.5 and homozygote TNC expressing littermates at Day E12.5.

\section{Branching Morphogenesis and Growth}

In our current study we characterized lung branching morphogenesis (1) by the number of branches occurring (called branching) and (2) by the pattern of airway branching (called branching pattern). The number of terminal endbuds was taken as a measure of branching. After $48 \mathrm{~h}$ in culture homozygote TNC-deficient explants showed a highly sig-

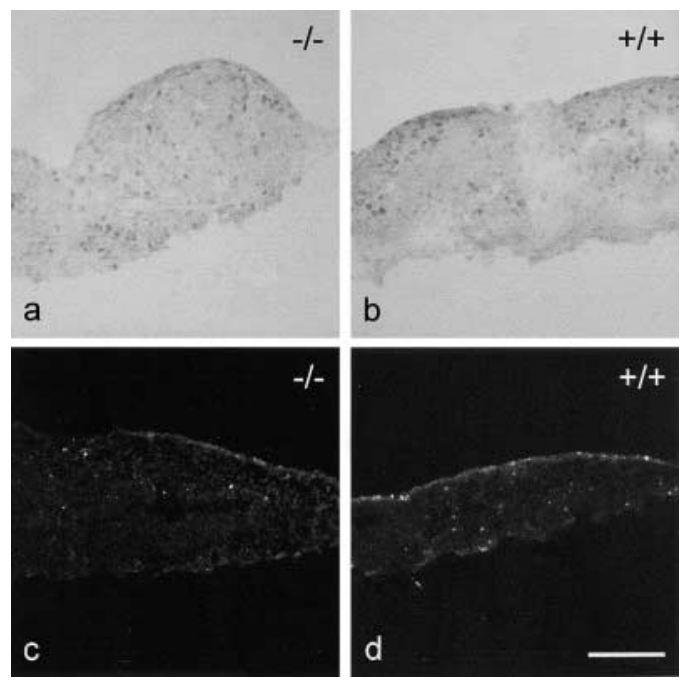

Figure 5. Cell proliferation and cell death. After $54 \mathrm{~h}$ in culture explants were cross-sectioned and stained for cell proliferation (mAB MIB-5, $a$ and $b$ ), or programmed cell death (TUNEL, $c$ and $d$ ), respectively. No difference between homozygote TNCdeficient $(a$ and $c ;-/-)$ and wild-type $(b$ and $d ;+/+)$ explants was observed.

nificant reduction of the number of endbuds as compared with wild-type $(36 \%, P<0,001$, start of culture $=$ E12.5). In lung explants of heterozygote TNC-deficient fetuses the number of the terminal endbuds was also reduced, but only half of the reduction mentioned above was observed (18\%, $P<0.002$; Figures 1 and 2, Table 1). Similar results were obtained using lungs which were explanted at Day E11.5 (Table 1). Comparing the growth of the explants, we did not detect any significant difference between TNC null and wild-type explants (Figures 1 and 2). However, we observed an enlargement of the size of the terminal endbuds in the TNC-null explants (Figures 1 and 3). The latter results were confirmed by morphometric measurements (Figure 4). Branching was followed for every individual terminal endbud between 15 and $40 \mathrm{~h}$ after explantation and their size was measured at the latter time point. In the group of "no branching" the size of the homozygote as well as of the heterozygote TNC-deficient endbuds was roughly twice as large as that in controls. The size of the homozygote TNCnull endbuds which had branched once was enlarged by $>20 \%$. In contrast to wild-type and heterozygote TNCdeficient explants, the homozygote TNC-null explants did not contain any endbud that branched twice during the period of observation (Figure 4).

In vivo the branching pattern of the larger airways appears to be very constant (31). We also observed this in our wild-type lung organ cultures, when whole lungs were explanted at Day E11.5. As shown in Figure 3, TNC-null explants showed the same very constant branching pattern as the wild type up to the time point $(\sim 3 \mathrm{~d}$ in culture) where the bronchial tree became too complex to be compared.

\section{Cell Proliferation and Cell Death}

Wondering if the balance between cell proliferation and cell death may explain the observed lung phenotype of the 


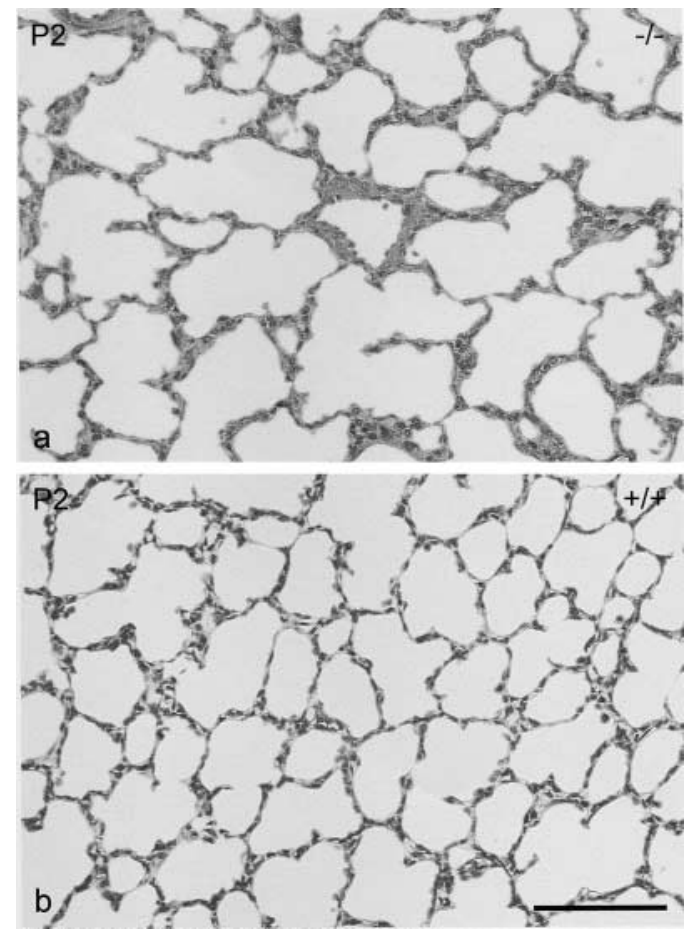

Figure 6. Lung morphology at Postnatal Day 2. Comparing the morphology of TNC-null lungs $(a ;-/-)$ to wild-type lungs ( $b$, $+/+)$ at Postnatal Day $2(\mathrm{P} 2)$, the observed airspaces of the gas exchange area appeared larger in TNC-deficient lungs than the wild-type ones. Scale bar: $100 \mu \mathrm{m}$.

TNC null mice, we stained cross-sections of the explants for cell proliferation (mAB MIB-5), or programmed cell death (TUNEL), respectively, at Day E11.5 (four explants of each condition). No difference between homozygote TNC-deficient and wild-type explants could be observed (Figure 5).

\section{Lung Morphology at Postnatal Day 2}

We studied the lung morphology at Postnatal Day 2 to support our in vitro data with in vivo observations. Day P2 was chosen because at this time point branching morphogenesis is completed and alveolarization has not yet started

TABLE 1

Number of used explants and observed number of branches

\begin{tabular}{lccrcr}
\hline & \multicolumn{3}{c}{ E11.5 } & & \multicolumn{2}{c}{ E12.5 } \\
\cline { 2 - 3 } \cline { 5 - 6 } & No. of Explants & $\%$ Branching & & No. of Explants & $\%$ Branching \\
\hline TNC $-/-$ & 9 & $54 \pm 1.8^{* * *}$ & 8 & $64 \pm 5.6^{* * *}$ \\
TNC $+/-$ & - & - & & 4 & $82 \pm 4.7^{*}$ \\
TNC $+/+$ & 8 & $100 \pm 13.5$ & 13 & $100 \pm 7.1$ \\
\hline
\end{tabular}

Lungs were obtained from fetuses at Embryonic Days E11.5 or E12.5, respectively. The number of terminal endbuds was counted after two days in culture and normalized to the controls. The standard deviation and the $P$ value of the Student's $t$ test were calculated.

$* P<0.002$

** $P<0.001$.
TABLE 2

Total and specific lung volume

\begin{tabular}{ccc}
\hline & \multicolumn{2}{c}{ P2 } \\
\cline { 2 - 3 } & Total Lung Volume & Specific Lung Volume \\
\hline TNC $-/-$ & $0.084 \pm 0.015 \mathrm{ml}$ & $0.047 \pm 0.005 \mathrm{ml} / \mathrm{g}$ body weight \\
TNC $+/+$ & $0.076 \pm 0.060 \mathrm{ml}$ & $0.045 \pm 0.008 \mathrm{ml} / \mathrm{g}$ body weight
\end{tabular}

No significant difference was observed between the lung volumes of TNCdeficient and wild-type animals $(P \geqslant 0.35)$. Means of three animals per group and standard deviations are given.

(3). Therefore, we were able to investigate the end point of branching morphogenesis. We did not study any earlier time point, because it would not have been possible to distinguish between reduced or delayed branching. The total and the specific lung volumes of TNC null and wildtype mice at Day P2 did not show any significant difference (Table 2). But comparing the morphology of the lung parenchyma of TNC-deficient and wild-type lungs, we observed an enlargement of the airspaces of the gas exchange area in TNC-null lungs (Figure 6). This result was verified by estimating the mean volume-weighted airspace volume. In TNC-deficient lungs the airspace volume was estimated to be almost two times (factor 1.85) larger than in wild-type lungs. Because we did not detect any other morphologic differences between knockout and wild type, the enlarged airspaces may be taken as an in vivo confirmation of the reduced branching observed in TNC-null lung organ cultures.

\section{Discussion}

Despite the fact that many in vitro studies attributed a developmental role to TNC, no developmental phenotype was described in TNC-deficient mice until now (see above; $11,13)$. Using lung organ cultures we were able to show that the number of branches was reduced by $36-46 \%$ in homozygote TNC-deficient lung explants as compared with wild-type controls (Figures 1-3, Table 1). In heterozygote TNC-deficient lung explants half of the reduction was observed (Figures 1 and 2, Table 1), predicting that not only the presence of TNC, but also its amount is important. These results represent the first mammalian TNC null phenotype, where a developmental process caused structural alterations.

However, it may be argued that lung organ cultures are only in vitro studies. Therefore, we investigated the lung at Postnatal Day P2. At this time point branching morphogenesis is completed and septation (alveolarization) has not yet started (3). Volumetric comparison of TNC-null and wild-type lungs did not reveal any significant difference for either total or specific lung volume (Table 2). Based on morphologic observations and on the estimation of the mean volume-weighted airspace volume, the airspaces of the lung parenchyma appeared to be almost two times larger in TNC-null lungs than in the wild-type ones (see REsults and Figure 6). Because we did not observe any alteration of the nonparenchymal structures in TNC-deficient lungs, we conclude that the enlarged airspaces of the TNC-null 
lungs represent the result of a reduced branching of the bronchial tree in the knockout mice. These morphologic in vivo observations are supported by our in vitro data. We measured the size of the terminal endbuds and were able to show that especially those endbuds were enlarged that did not branch during the observation period of $25 \mathrm{~h}$ (Figure 4). This result represents further evidence that TNC deficiency leads to an inhibition of branching, but not to an inhibition of growth of the epithelial tubules (Figures 1, 2 , and 4).

Our results reflect previously reported perturbation experiments using anti-TNC antibodies and bacterially expressed TNC fragments in rat lung organ cultures. Young and coworkers (16) described reduced branching, enlarged terminal buds, and unaltered explant growth after perturbation. Therefore, the lung represents one of the very few examples where the results of perturbation experiments correctly predicted a phenotype of the TNC-null mouse. One reason for this result may be that the lung represents one of the few organs in which TNC is more abundant than tenascin-X (7).

During branching morphogenesis TNC is expressed at the epithelial-mesenchymal interface and accumulates at the growing tips of the bronchial tree where airway subdivision takes place $(15,16,32)$. This zone is characterized by an intensive and specific epithelial cell proliferation and migration (33). As a multifunctional protein, TNC is involved in processes like cell proliferation and migration (13). Because both cell proliferation and cell death were not significantly altered in the developing TNC-null lungs (Figure 5), we hypothesize that TNC predominately influences cell migration, or more specifically the penetration of the growing bronchial tip into the surrounding mesenchyme. In a growing organ, cleft formation (formation of the branching point) is viewed as a spot where the epithelial cells of the growing tip stop moving, while their neighbors continue their penetration into the mesenchyme. Therefore, we further hypothesize that TNC also takes part in the control of cleft formation. This view is supported by our observation that the terminal ends of the bronchial tree are enlarged in TNC-null lungs throughout development (Figure 4).

Proper lung development is highly sensitive to mechanical stimuli and pressure. Fetal breathing movements $(34,35)$ and spontaneous contractions of the future airways are important for proper lung development $(23,36)$. A compression of the lung, as occurred in oligohydraneous fetuses, impairs lung development (37). The expression of TNC at the terminal ends of the growing bronchial tree correlates with mechanical stimuli $(15,16,32)$. Both fetal breathing movements (38) and spontaneous contractions of the future airways (23) are extending the terminal buds rhythmically. Our observed reduction of the number of branches in TNCnull lungs lets us speculate that TNC may be one of the factors transducing mechanical stimuli into cellular behavior.

In summary, we were able to describe the first developmental phenotype of the TNC-null mouse that causes structural alterations of an organ. Most likely TNC contributes to lung branching morphogenesis by the control of cell migration during growth of the terminal endbuds and during cleft formation. Furthermore, it may take part in the transduction of important mechanical stimuli acting on the termiends of the growing bronchial tree.

Acknowledgments: The authors thank Drs. Reinhard Fässler and Peter H. Burri for continuous support and helpful discussions, and Mrs. Marianne Hofstetter, Mrs. Catherine Allemann-Probst, and Mrs. Bettina de Breuyn for expert technical assistance. This work was supported by grants of the Swiss National Science Foundation (no. 3100.55895 .98 to Peter H. Burri and no. 3100.068256 .02 to J.S.), of the Max Planck Society (Reinhard Fässler), and of the Deutsche Forschungsgemeinschaft (Reinhard Fässler), and by the Fonds de perfectionnement du CHUV, Lausanne (M.R.-K.).

\section{References}

1. Spooner, B. S., and N. K. Wessells. 1970. Mammalian lung development: interactions in primordium formation and bronchial morphogenesis. J. Exp. Zool. 175:445-454.

2. Sekine, K., H. Ohuchi, M. Fujiwara, M. Yamasaki, T. Yoshizawa, T. Sato, N. Yagishita, D. Matsui, Y. Koga, N. Itoh, and S. Kato. 1999. Fgf10 is essential for limb and lung formation. Nat. Genet. 21:138-141.

3. Burri, P. H. 1999. Lung development and pulmonary angiogenesis. In Lung Disease. C. Gaultier, J. Bourbon, and M. Post, editors. Oxford University Press, New York. 122-151.

4. Ten Have-Opbroek, A. A. W. 1991. Lung development in the mouse embryo. Exp. Lung Res. 17:111-130.

5. Mackie, E. J., W. Halfter, and D. Liverani. 1988. Induction of tenascin in healing wounds. J. Cell Biol. 107:2757-2767.

6. Talts, J. F., G. Wirl, M. Dictor, W. J. Muller, and R. Fässler. 1999. Tenascin-C modulates tumor stroma and monocyte/macrophage recruitment but not tumor growth or metastasis in a mouse strain with spontaneous mammary cancer. J. Cell Sci. 112:1855-1864.

7. Geffrotin, C., J. J. Garrido, L. Tremet, and M. Vaiman. 1995. Distinct tissue distribution in pigs of tenascin-X and tenascin-C transcripts. Eur. J. Biochem. 231:83-92.

8. Matsumoto, K., Y. Saga, T. Ikemura, T. Sakakura, and R. Chiquet-Ehrismann. 1994. The distribution of tenascin-X is distinct and often reciprocal to that of tenascin-C. J. Cell Biol. 125:483-493.

9. Weber, P., D. Montag, M. Schachner, and R. R. Bernhardt. 1998. Zebrafish tenascin-W, a new member of the tenascin family. J. Neurobiol. 35:1-16.

10. Hagios, C., M. Brown-Luedi, and R. Chiquet-Ehrismann. 1999. Tenascin-Y, a component of distinctive connective tissues, supports muscle cell growth. Exp. Cell Res. 253:607-617.

11. Mackie, E. J., and R. P. Tucker. 1999. The tenascin-C knockout revisited. J. Cell Sci. 112:3847-3853.

12. Jones, F. S., and P. L. Jones. 2000. The tenascin family of ECM glycoproteins: structure, function, and regulation during embryonic development and tissue remodeling. Dev. Dyn. 218:235-259.

13. Jones, P. L., and F. S. Jones. 2000. Tenascin-C in development and disease: gene regulation and cell function. Matrix Biol. 19:581-596.

14. Koch, M., B. Wehrle, S. Baumgartner, J. Spring, D. Brubacher, and M. Chiquet. 1991. Epithelial synthesis of tenascin at tips of growing bronchi and graded accumulation in basement membranes and mesenchyme. Exp. Cell Res. 194:297-300.

15. Zhao, Y., and S. L. Young. 1995. Tenascin in rat lung development: in situ localization and cellular sources. Am. J. Physiol. 13:L482-L491.

16. Young, S. L., L.-Y. Chang, and H. P. Erickson. 1994. Tenascin-C in rat lung: distribution, ontogeny and role in branching morphogenesis. Dev. Biol. 161:615-625

17. Saga, Y., T. Yagi, Y. Ikawa, T. Sakakura, and S. Aizawa. 1992. Mice develop normally without tenascin. Genes Dev. 6:1821-1831.

18. Forsberg, E., E. Hirsch, L. Frohlich, M. Meyer, P. Ekblom, A. Aszodi, S. Werner, and R. Fassler. 1996. Skin wounds and severed nerves heal normally in mice lacking tenascin-C. Proc. Natl. Acad. Sci. USA 93:65946599.

19. Fukamauchi, F., Y. J. Wang, N. Mataga, and M. Kusakabe. 1997. Paradoxical behavioral response to apomorphine in tenascin-gene knockout mouse. Eur. J. Pharmacol. 338:7-10.

20. Ohta, M., T. Sakai, Y. Saga, S. Aizawa, and M. Saito. 1998. Suppression of hematopoietic activity in tenascin-C-deficient mice. Blood 91:4074-4083.

21. Matsuda, A., A. Yoshiki, Y. Tagawa, H. Matsuda, and M. Kusakabe. 1999 Corneal wound healing in tenascin knockout mouse. Invest. Ophthalmol. Vis. Sci. 40:1071-1080.

22. Matsumoto, K., N. Hiraiwa, A. Yoshiki, M. Ohnishi, and M. Kusakabe. 2002. PDGF receptor-alpha deficiency in glomerular mesangial cells of Tenascin-C knockout mice. Biochem. Biophys. Res. Commun. 290:12201227.

23. Schittny, J. C., G. Miserocchi, and M. P. Sparrow. 2000. Spontaneous peristal- 
tic airway contractions propel lung liquid through the bronchial tree of intact and fetal lung explants. Am. J. Respir. Cell Mol. Biol. 23:11-18.

24. Howard, C. V., and M. G. Reed. 1998. Unbiased Stereology: Three-Dimensional Measurement in Microscopy. Springer Verlag, Berlin, Heidelberg, New York. 143-145.

25. Luyet, C., P. H. Burri, and J. C. Schittny. 2002. Suppression of cell proliferation and programmed cell death by dexamethasone during postnatal lung development. Am. J. Physiol. Lung Cell. Mol. Physiol. 282:477-483.

26. Schittny, J. C., V. Djonov, A. Fine, and P. H. Burri. 1998. Programmed cell death contributes to postnatal lung development. Am. J. Respir. Cell Mol. Biol. 18:786-793.

27. Ito, T., H. Mitui, N. Udaka, H. Hayashi, K. Okudela, M. Kanisawa, and H. Kitamura. 1998. Ki-67 (MIB 5) immunostaining of mouse lung tumors induced by 4-nitroquinoline 1-oxide. Histochem. Cell Biol. 110:589-593.

28. Gerdes, J., H. Lemke, H. Baisch, H. H. Wacker, U. Schwab, and H. Stein. 1984. Cell cycle analysis of a cell proliferation-associated human nuclear antigen defined by the monoclonal antibody Ki-67. J. Immunol. 133:1710 1715 .

29. Scherle, W. 1970. A simple method for volumetry of organs in quantitative stereology. Mikroskopie 26:57-60.

30. Weibel, E. R. 1970. Morphometric estimation of pulmonary diffusion capacity: I. Model and method. Respir. Physiol. 11:54-75.
31. Kaufmann, M. H. 1995. The Atlas of Mouse Development. Academic Press, San Diego, London.

32. Kaarteenaho-Wiik, R, V. Kinnula, R. Herva, P. Pääkkö, R. Pöllänen, and Y. Soini. 2001. Distribution and mRNA expression of Tenascin-C in developing human lung. Am. J. Respir. Cell Mol. Biol. 25:341-346.

33. Mollard, R., and M. Dziadek. 1998. A correlation between epithelial proliferation rates, basement membrane component localization patterns, and morphogenetic potential in the embryonic mouse lung. Am. J. Respir. Cell Mol. Biol. 19:71-82.

34. Tseng, B., S. Cavin, F. Booth, E. Olson, M. Marin, T. McDonnell, and I. Butler. 1999. Pulmonary hypoplasia in the myogenin null mouse embryo. Am. J. Respir. Cell Mol. Biol. 22:304-315.

35. Kitterman, J. 1996. The effects of mechanical forces on fetal lung growth. Clin. Perinatol. 23:727-740.

36. Roman, J. 1995. Effects of calcium channel blockade on mammalian lung branching morphogenesis. Exp. Lung Res. 21:489-502.

37. Moessinger, A., M. Singh, D. Donnelly, G. Haddad, M. Collins, and L. James. 1987. The effect of prolonged oligohydramnios on fetal lung development, maturation and ventilatory patterns in the newborn guinea pig. J. Dev. Physiol. 9:419-427.

38. Harding, R. 1997. Fetal pulmonary development: the role of respiratory movements. Equine Vet. J. 24:32-39. 